\title{
Smoking status ascertainment and interventions in acute medical patients
}

\author{
R Murray, ] Leonardi-Bee, ] Marsh, L Jayes and J Britton
}

\begin{abstract}
Hospital admission provides an opportunity to promote smoking cessation. Clinical guidelines recommend ascertainment of smoking status and delivery of cessation interventions in all consultations. In this article, smoking ascertainment and intervention among all patients admitted to medical wards in a UK hospital over a four-week period in 2010 were audited. Medical records of 767 patients were screened; 96 (13\%) were current smokers, 243 (32\%) ex-smokers and 233 (30\%) non-smokers. There was no record of smoking status in 243 (25\%) individuals and this proportion varied between specialties. Of the 96 current smokers, only 23 received documented cessation advice or pharmacological support. Four weeks after discharge, $31 \%$ reported that they were abstinent from smoking, representing $50 \%$ of those who received support and $20 \%$ of those who did not. Ascertainment of smoking status and delivery of cessation support to patients admitted to medical wards was low, suggesting that there is room for improvement in the management of smoking among inpatients.
\end{abstract}

\section{KEY WORDS: cessation support, recording, smoking}

\section{Introduction}

Tobacco smoking is the largest avoidable cause of premature death and disability in the world, ${ }^{1}$ and helping smokers to quit smoking is one of the most cost-effective interventions available in medicine. ${ }^{2}$ It is therefore important that smokers are identified and offered help to stop smoking, and systematic integration of smoking ascertainment and provision of cessation interventions into healthcare services has been recommended in the UK by clinical guidelines first published in $1998,{ }^{3}$ and by National Institute for Health and Clinical Excellence (NICE) guidance since $2006 .{ }^{4}$

Hospital admission is a major opportunity to promote smoking cessation, ${ }^{5}$ and all patients admitted to hospital should have smoking status recorded and, if appropriate, be provided with cessation pharmacotherapy and behavioural support. ${ }^{3,4}$ However, while objective evidence on the provision of cessation

R Murray, lecturer in health policy and promotion; ] Leonardi-Bee, associate professor in medical statistics; J Marsh, research associate; L Jayes, research associate; J Britton, professor of epidemiology and consultant respiratory physician

Division of Epidemiology and Public Health and UK Centre for Tobacco Control Studies, University of Nottingham, Clinical Sciences Building, Nottingham City Hospital support in UK hospitals is scant, anecdotal evidence suggests that delivery is at best inconsistent. It is therefore likely that few of the estimated four million smokers admitted to hospital in the UK each year receive effective cessation support during their admission. The present study was therefore designed to audit the level of smoking status recording and delivery of cessation advice on medical wards in one UK hospital.

\section{Methods}

The smoking status ascertainment and cessation intervention was audited in all discharges from the 18 specialist medical wards at Nottingham City Hospital between 6 September and 1 October 2010. Patients ready for discharge within 24 hours (or on Fridays, within 72 hours) were identified by visiting each ward every day from Monday to Friday, and by consulting inpatient lists and checking with nursing staff and/or discharge managers. Clinical inpatient notes for these individuals were then searched for documentation of smoking status during the current hospital admission, either in an admission proforma checklist, or in free text records. The admission proforma allowed patients to be categorised as 'current smokers', 'ex-smokers' or those who had 'never smoked'. Notes were also searched for evidence that smoking cessation advice had been delivered to smokers and for prescription of pharmacotherapy given during admission and/or at discharge.

All ex-smokers and patients with no record of smoking status were interviewed before leaving hospital to determine whether they had smoked within 12 months of hospital admission. Those who had, in addition to all documented current smokers, were asked to provide a sample of exhaled air for carbon monoxide measurement immediately before discharge, and to give consent for the study team to contact them one month after discharge to ask about current smoking status, uptake of stop smoking services, and any use of pharmacotherapy. Attempts were made to contact every patient who consented to follow up, either by telephone or email, around a month after discharge to ascertain smoking status. Participants who then reported that they were not smoking were seen to take a further measure of exhaled carbon monoxide. Non-smokers were defined as those with an exhaled carbon monoxide reading below 10 parts per million (ppm), ${ }^{6}$ and at least five attempts were made to contact all participants. Full ethics approval to contact patients at follow-up was granted by the Nottingham Research Ethics Committee. ${ }^{1}$

The number of patients recorded as having been discharged from the study wards during the study period according to routine hospital episode statistics was used to estimate the number 
of patients potentially eligible for involvement in the study. Data were analysed using SPSS for Windows (Version 16.0) and are summarised as means with standard deviations and ranges for continuous data, or as frequencies and percentages for dichotomous data.

\section{Results}

Of the total 836 patients recorded in routine hospital episode statistics records as having been discharged from the study wards during the study period, the medical records of 767 (92\%), of mean (SD) age 65.6 (18.5) years, and of whom $52 \%$ were male, were identified and screened. Most of the $69(8 \%)$ patients who were not included were discharged with less than 24 hours notice. Medical records included documentation that $96(12.5 \%)$ of the 767 study patients were current smokers, 243 (31.7\%) ex-smokers, and 233 (30.4\%) non-smokers. There was no record of smoking status in 195 (25.4\%) individuals. Breaking these figures down by medical specialty illustrates a wide range in the proportion with missing smoking status, from $15 \%$ of patients on endocrinology wards to over $50 \%$ of all patients on renal wards (Table 1). A chi squared test showed a highly statistically significant association between recording of smoking status and specialty of ward $(\mathrm{p}<0.001)$.

Of the 96 patients documented as current smokers on admission, delivery of any form of smoking cessation advice or support during the hospital stay or at discharge was recorded for 23 individuals, and varied between specialties from $0 \%$ on infectious diseases and renal wards to $37.5 \%$ on respiratory wards. Eleven of these smokers were prescribed nicotine replacement therapy (NRT) both at admission and discharge, and eight at discharge only. An offer of behavioural support was documented for six patients during the hospital stay, of whom one also received NRT at admission and discharge, three received NRT at discharge only, and two received no pharmacological support. Referral to NHS Stop Smoking services on discharge was documented for five patients, two of whom received NRT at both admission and discharge and three of whom received no documented support during their hospital stay. No patient was prescribed varenicline or bupropion at any time (Table 2). Our ascertainment shortly before discharge identified 115 smokers and recent ex-smokers (mean age (SD) 57.0 years (14.6); 59\% male), of whom 68 (mean age (SD) 57.4 years (13.3); 65\% male) consented to follow-up. Of these, $49(72 \%)$ were documented to be current smokers at admission, five (7\%) ex-smokers and 14 (21\%) had no record of smoking status at admission. At discharge, 36 were abstinent from smoking and considered themselves to have quit, and this was validated by $\mathrm{CO}$ measurement. A further 16 considered themselves to still be smokers but had CO readings below $10 \mathrm{ppm}$ with the remaining 16 having continued to smoke while in hospital and having $\mathrm{CO}$ readings above $10 \mathrm{ppm}$. Approximately half of those who consented for

\begin{tabular}{llc}
$\begin{array}{l}\text { Table 2. Smoking cessation advice and support offered to recorded } \\
\text { smokers. }\end{array}$ & \\
Smoking cessation advice given & Yes & $18(18.8 \%)$ \\
& Not recorded & $78(81.2 \%)$ \\
Cessation pharmacotherapy & NRT & $14(14.6 \%)$ \\
prescribed on admission* & Bupropion & $0(0 \%)$ \\
& Varenicline & $0(0 \%)$ \\
Behavioural support & None & $82(85.4 \%)$ \\
offered during admission?* & Yes & $6(6.2 \%)$ \\
Behavioural support accepted & Not recorded & $90(93.8 \%)$ \\
& Yes & $3(50 \%)$ \\
Prescription on discharge* & No & $3(50 \%)$ \\
& NRT & $19(19.8 \%)$ \\
& Bupropion & $0(0 \%)$ \\
Referral to stop smoking & Varenicline & $0(0 \%)$ \\
service on discharge* & None & $77(80.2 \%)$ \\
\hline *Groups are not mutually exclusive. & Yes & $5(5.2 \%)$ \\
NRT= nicotine replacement therapy. & & $91(94.8 \%)$ \\
& Not recorded & \\
\hline
\end{tabular}

Table 1. Smoking status recording overall, and by medical specialty.

\begin{tabular}{|c|c|c|c|c|}
\hline & Current smoker $n(\%)$ & Ex-smoker n(\%) & Non-smoker n(\%) & No smoking status recorded $n(\%)$ \\
\hline Overall & $96(12.5)$ & $243(31.7)$ & $233(30.4)$ & $195(25.4)$ \\
\hline \multicolumn{5}{|l|}{ Specialty: } \\
\hline Endocrinology & $13(10.2)$ & $47(36.7)$ & $49(38.3)$ & $19(14.8)$ \\
\hline Respiratory & $24(18.0)$ & $63(47.4)$ & $25(18.8)$ & $21(15.8)$ \\
\hline Oncology & $16(15.4)$ & $37(35.6)$ & $30(28.8)$ & $21(20.2)$ \\
\hline Stroke & $13(11.3)$ & $35(30.5)$ & 39 (33.9) & $28(24.3)$ \\
\hline Infectious disease & $3(8.6)$ & $4(11.4)$ & $19(54.3)$ & $9(25.7)$ \\
\hline Cardiac & $10(11.1)$ & $30(33.3)$ & $24(26.7)$ & $26(28.9)$ \\
\hline Haematology & $13(13.5)$ & $19(19.8)$ & $28(29.2)$ & $36(37.5)$ \\
\hline Renal & $4(6.2)$ & $8(12.3)$ & $18(27.7)$ & 35 (53.8) \\
\hline
\end{tabular}


follow-up, and were recorded as smokers at admission, were $\mathrm{CO}$-validated as current non-smokers at the time of discharge; likewise half of those who had no smoking status recorded at admission were $\mathrm{CO}$ validated as non-smokers at the time of discharge with the other half being current smokers.

\section{Follow-up}

Four weeks after discharge, 21 patients reported that they were abstinent from smoking and 18 were $\mathrm{CO}$ validated as such; the remaining three declined a follow-up visit for CO measurement. Seventeen of those who were abstinent from smoking at four weeks were recorded as current smokers on admission to hospital. Of the other 47 who agreed to follow-up assessment, 28 reported that they were smoking and four had died. Fifteen were unreachable $(22.1 \%)$. Only $15(25 \%)$ patients had used NRT and $13(19 \%)$ had used the stop smoking services after discharge. Only three of the patients who used support after discharge had received NRT while in hospital; the remainder had no documentation of any form of smoking cessation advice or support in their medical notes (Table 3).

\section{Relation between use of support and quit status}

A chi-squared test showed that there was a statistically significant association between the use of NRT or accessing services and quit status at four weeks after discharge $(\mathrm{p}=0.02)$; where $50 \%$ of those who used NRT after discharge or accessed services quit as compared to only $21.7 \%$ of those who did not use NRT after discharge or access services (odds ratio 3.6, 95\% confidence interval 1.21 to 10.71$)$. Those who had died or were noncontactable are assumed to be still smoking at follow-up.

\section{Discussion}

This study shows that ascertainment of smoking status in admissions to acute medical wards in this hospital is low, at an average of $75 \%$, with the delivery of smoking cessation advice

Table 3. Smoking status and sources of support used in quit attempts at four weeks after discharge.

\begin{tabular}{llc} 
Outcome at four weeks & Quit & $21(30.9 \%)$ \\
& Still smoking & $28(41.2 \%)$ \\
& Not contactable & $15(22.1 \%)$ \\
& Died & $4(5.9 \%)$ \\
Validated smoking & Validated & $18(85.7 \%)$ \\
status (of quitters) & Self report & $3(14.3 \%)$ \\
NRT used after discharge & Yes & $17(25 \%)$ \\
& No & $51(75 \%)$ \\
Stop smoking services & Yes & $13(19.1 \%)$ \\
accessed after discharge & No & $55(80.9 \%)$ \\
\hline NRT= nicotine replacement therapy. & \\
& &
\end{tabular}

and/or support being very low at less than $20 \%$ of all reported smokers. In the majority of cases, this support consisted of NRT alone; only six patients were offered behavioural support during their hospital stay, and five referred to stop smoking services on discharge. No patient was prescribed either varenicline or bupropion, and smoking status recording varied widely between specialties. Of smokers and recent ex-smokers who consented to follow up, less than a third (31\%) were abstinent from smoking one month after discharge. Abstinence was significantly more likely among those who received support.

Current NICE guidance, which should define standard hospital practice, indicates that all smokers admitted to hospital should be offered help to quit smoking. The cessation support available in this hospital at the time of the study is probably typical of many UK hospitals, in that it follows NICE guidance to a minimum level; that is, there is no specialist cessation service available on site, and intervening in smoking is left to the discretion and initiative of medical staff. Some wards had established systematic approaches to ensure that patients who wanted to quit smoking were referred on to NHS Stop Smoking Services in the community at the point of discharge, typically as a written list of referral details, but this was not consistently employed on these wards; most had no such system and relied on the initiative of junior medical or nursing staff to arrange follow-up. It is possible that this study has underestimated the true proportion of smokers offered cessation support since it was possible to only measure documented offers and hence cases in which verbal offers were made but declined and not recorded may have been omitted. However, the extremely low levels of cessation medication use observed suggest that a great deal more might be done.

The service at this hospital relies on the medical team identifying smokers, prescribing NRT or pharmacotherapy where appropriate, and referral to support services on discharge, since there is no dedicated smoking cessation service on site. One of the possible reasons for the low figures found in this audit is a lack of knowledge on the part of the ward staff. While it should be expected that clinicians give brief advice to quit to all patients, local arrangements for referral to smoking cessation services differ and, in many cases, junior doctors in particular may be unaware of how to refer patients in to formal cessation services. If this truly is the case then education of clinical staff as to how to offer brief advice and refer into services needs to be considered, however it should not be a reason for not ascertaining smoking status.

A small number of participants received smoking cessation support after discharge from hospital but the majority received no help at all. These findings apply to only one UK hospital and could therefore be atypical of national experience, but there is no evidence that this is in fact the case. This study identifies gross under-estimation and under-management of smoking among hospital inpatients that is likely to be more widespread than the single hospital involved. The population of Nottingham City (which is served by the hospital involved in this audit) is just over 300,000 and a smoking prevalence of 
$32 \%$, one the highest smoking rates for local authorities in England. Given the latter statistic, it would be reasonable to assume that tackling smoking rates in any given setting should be a priority for the local healthcare system, thus again indicating that an under-estimation of the UK picture from this study is unlikely. Marked differences in ascertainment of smoking status between medical specialties were also identified. This could be due in part to previous ascertainment of smoking status and refusal of cessation support in specialties where readmissions are relatively common, for example renal medicine, but raises the concern that, if so, smokers whose motivation has changed are being missed. Clinical guidelines recommend that this information is collected at each contact to ensure that smokers who are motivated to quit are supported in doing so.

At one month after discharge only $31 \%$ of smokers and recent ex-smokers were abstinent from smoking, which is substantially less than the $49 \%$ cessation achieved by smokers attending NHS Stop Smoking Services. ${ }^{7}$ This figure includes a number who reported that they were ex-smokers on admission, though on the assumption that many of these will have quit smoking either immediately prior to admission, or at the onset of the illness which precipitated admission, recent ex-smokers were included on the grounds that they were probably more likely to relapse into smoking than more established ex-smokers. However, the relatively low realised quit rate reflects a failure to deliver adequate cessation support. It is also possible that appropriate support was not offered to patients who wrongly self-reported as 'never smokers'. The results have illustrated that those patients who used NRT or smoking cessation services were significantly more likely to quit than those who did not, supporting the notion that intervening in the hospital setting is an effective means of increasing quit rates and highlighting the need for accurate recording of current smoking status in order to identify those patients who should be offered support.

Research into the provision of cessation services in secondary care to date has tended to focus on the content of smoking cessation services, rather than methods of making sure that delivery happens. Thus, while a model content of secondary care services has been defined, ${ }^{8}$ delivery is far from optimal, ${ }^{5,9,10}$ especially when delegated to busy general staff with many other conflicting demands on their time. ${ }^{11}$ These findings are reinforced by the current study and highlight the requirement for smoking status to be recorded for all new hospital admissions and for appropriate cessation support to then be offered. Nearly half a million hospital admissions each year are attributable to smoking, ${ }^{12}$ and so there is a clear need to reduce smoking prevalence, and thus the financial burden on the NHS. The point at which a smoker is admitted to hospital is a prime opportunity to intervene with smoking cessation support, ${ }^{5}$ not least because the integration of smoking cessation interventions into healthcare services has been recommended. ${ }^{3,4}$ There is a clear need for further research investigating ways of delivering smoking cessation support in this setting to ensure delivery is comprehensive.

\section{Acknowledgements}

This article presents independent research commissioned by the National Institute for Health Research (NIHR) under its Programme Grants for Applied Research funding scheme (RP-PG-0608-10020). The views expressed in this article are those of the author(s) and not necessarily those of the NHS, the NIHR or the Department of Health.

\section{References}

1 Lowey H, Tocque K, Bellis MA, Fullard B. Smoking cessation services are reducing inequalities. J Epidemiol Comm Health 2003;57:579-80.

2 Tengs TO, Adams ME, Pliskin JS et al. 500 lifesaving interventions and their cost-effectiveness. Risk Analysis 1995;15:369-90.

3 Raw M, McNeill A, West R. Smoking cessation guidelines for health professionals - a guide to effective smoking cessation interventions for the health care system. Thorax 1998;53:S1-S19.

4 National Institute for Health and Clinical Excellence. Brief interventions and referral for smoking cessation in primary care and other settings. London: NICE, 2006.

5 Freund M, Campbell E, Paul C et al. Smoking care provision in hospitals: a review of prevalence. Nicotine Tob Res 2008;10:757-74.

6 Jarvis MJ, Tunstallpedoe H, Feyerabend C, Vesey C, Saloojee Y. Comparison of tests used to distinguish smokers from nonsmokers. Am J Public Health 1987;77:1435-8.

7 Statistics on NHS Stop Smoking Services in England, April 2009 to March 2010; 2010.

8 Rigotti NA, Munafo MR, Stead LF. Smoking cessation interventions for hospitalized smokers: a systematic review. Arch Intern Med 2008;168:1950-60.

9 Kava T, Taylor J, Gamble E, Partridge MR. The availability of smoking cessation advice from health professionals - a census from one East London District. Respir Med 2000;94:983-4.

10 Hunt M, Kouimtsidis C, Reynolds M, Lind J, Ghodse H. Smoking amongst patients admitted to a general hospital: a missed opportunity for early intervention. Drugs Educ Prevent Policy 2003;1:31-8.

11 Smith PM, Reilly KR, Houston Miller N, DeBusk RF, Taylor CB. Application of a nurse-managed inpatient smoking cessation program. Nicotine Tob Res 2002;4:211-22.

12 The NHS Information Centre. Statistics on smoking: England, 2010. London: Department of Health, 2010.

Address for correspondence: Dr R Murray, Division of Epidemiology and Public Health and UK Centre for Tobacco Control Studies, University of Nottingham, Clinical Sciences Building, Nottingham City Hospital, Nottingham NG5 1PB. Email: Rachael.Murray@nottingham.ac.uk 